\title{
COPIGMENTAÇÃO INTRA E INTERMOLECULAR DE ANTOCIANINAS: UMA REVISÃO
}

\author{
LEILA D. FALCÃO * \\ DENISE M. BARROS ** \\ CONY GAUCHE ** \\ MARILDE T. BORDIGNON LUIZ ***
}

\begin{abstract}
Este trabalho apresenta revisão de literatura sobre a reação de copigmentação intra e intermolecular e sua relevância na estabilização de antocianinas. Flavonóides não-antociânicos, alcalóides, aminoácidos e nucleosídios, entre outros, podem atuar como copigmentos de antocianinas. $\mathrm{O}$ aumento na estabilidade das antocianinas ocorre devido à proteção fornecida pelo copigmento frente à reação de hidratação do cátion flavilium. Estudos ainda são necessários para avaliar a estabilidade de antocianinas adicionadas de copigmentos em sistemas modelos de alimentos, visando aumentar o espectro de aplicação dessas como corantes em alimentos e bebidas.
\end{abstract}

PALAVRAS-CHAVE: COPIGMENTAÇÃO; ANTOCIANINAS.

\section{INTRODUÇÃO}

As antocianinas, da família dos flavonóides, constituem grupo de pigmentos solúveis em água responsável pela maioria das cores vermelha, laranja e azul de flores, frutas e vegetais (BROUILLARD, 1983). São substâncias fenólicas, glicosídios de antocianidinas, polihidroxi derivadas do cátion flavilium (FIGUEIREDO et al., 1996).

* $\quad$ Mestre em Ciência dos Alimentos, Departamento de Ciência e Tecnologia de Alimentos, Centro de Ciências Agrárias (CCA), Universidade Federal de Santa Catarina (UFSC), Florianópolis (e-mail: leilafalcao@hotmail.com).

** Bolsistas Iniciação Científica PIBIC/CNPq - CAL/CCA/UFSC.

*** Professora, Doutora do Programa de Pós-Graduação de Ciência dos Alimentos CAL/CCA/UFSC (e-mail: bordign@cca.ufsc.br). 
Apresentam-se sob a forma de diferentes estruturas em equilíbrio, com predominância do cátion flavilium (vermelho) somente em condições ácidas (SARNI-MANCHADO et al., 1996) (Figura 1).

\section{FIGURA 1 - ESTRUTURAS DE ANTOCIANINAS EM EQUILÍBRIO EM MEIO AQUOSO ÁCIDO}

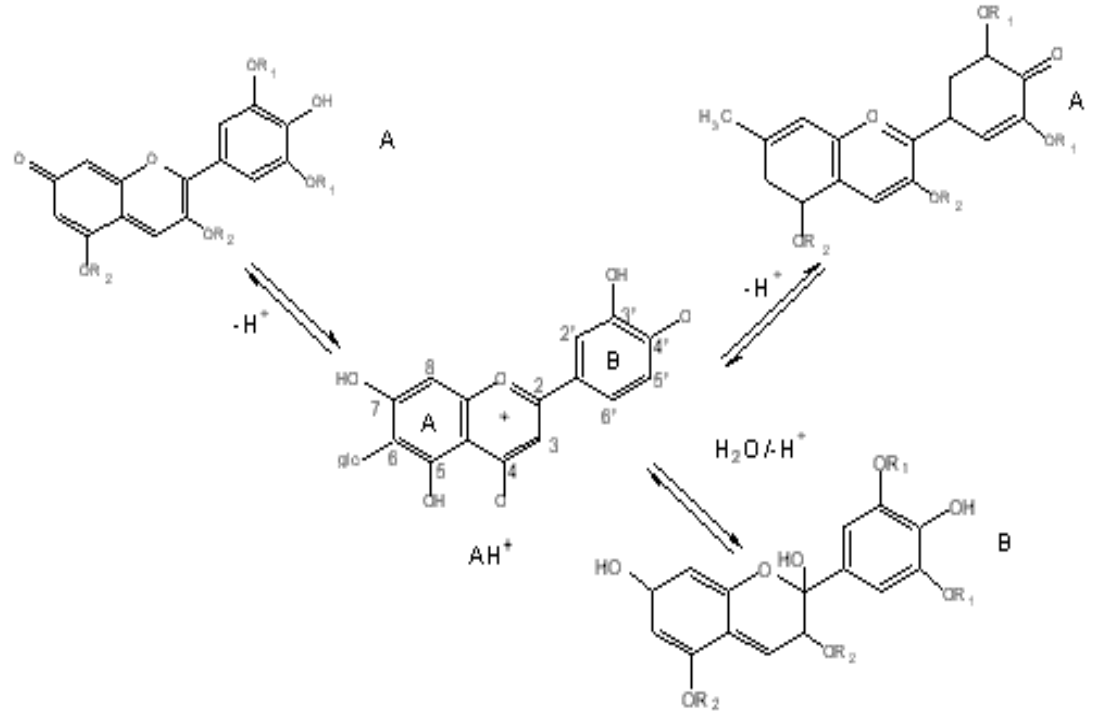<smiles></smiles><smiles>C=C=C</smiles>

$C E$

C2

A: bases quinoidais.

B: hemiacetal ou pseudobase carbi-nol. $\mathrm{AH}+$ : cátion flavilium. CE e CZ:

Chalconas.

$\mathrm{R} 1: \mathrm{H}, \mathrm{CH} 3$

R2: H, CH3, açúcar, açúcar acilado.

Fonte: FIGUEIREDO et al., 1996. 
A exigência do consumidor quanto à qualidade dos alimentos, bem como ações legislativas para redução do uso de corantes sintetizados quimicamente em alimentos, têm aumentado o interesse pelo desenvolvimento de corantes de fontes naturais (SAONA-RODRIGUEZ, GIUSTI e WROLSTAD, 1998). Para o suprimento da cor vermelha, as antocianinas representam alternativa atrativa (SARNI-MANCHADO et al., 1996) pelas cores brilhantes que fornecem. Além disso, apresentam atividades anticarcinogênicas (HAGIWARA et al., 2001, KAPADIA et al., 1997), antioxidantes (WANG et al., 2000; YOUDIM, MARTIN e JOSEPH, 2000) e antivirais (KAPADIA et al., 1997) reconhecidas cientificamente. Entretanto, a utilização de antocianinas nas indústrias de alimentos e de cosméticos ainda é restrita devido à sua baixa estabilidade em meios aquosos e pH acima de 2,0. Essa desvantagem e a percepção do consumidor de que pigmentos naturais são mais saudáveis geraram vários estudos. Tais pesquisas relatam aumento na estabilidade das antocianinas pelas ligações $O$-glicosídicas, presença de grupos acil na molécula de açúcar (FRANCIS, 1989), presença de íons metálicos (STRINGHETA,1991), auto-associação (BOULTON, 2001, MAZZA e BROUILLARD, 1987), inclusão molecular (PROVENZI, 2001; TAMURA et al., 1998; DANGLES, WIGAND e BROUILLARD, 1992) e copigmentação (AUBERT, DANGLES e AMIOT, 2001; DIMITRIC-MARKOVIC, PETRANOVIC e BARANAC, 2000; BARANAC, PETRANOVIC e DIMITRIC-MARKOVIC, 1996; KORZUCHOWSKA e WILSKA, 1996; DANGLES e BROUILLARD, 1992).

Este trabalho apresenta revisão bibliográfica sobre a reação de copigmentação inter e intramolecular e sua relevância na estabilização de antocianinas.

\section{COPIGMENTAÇÃO DAS ANTOCIANINAS}

$\mathrm{Na}$ natureza, moléculas de antocianinas estão freqüentemente associadas com moléculas incolores (copigmentos) que exercem efeitos determinantes sobre a cor dos vegetais (KORZUCHOWSKA e WILSKA, 1996). Na faixa de $\mathrm{pH}$ ligeiramente ácida para neutra, em que se enquadra grande parte dos vegetais, as antocianinas apresentam-se incolores (GONNET, 1998). Entretanto, as antocianinas encontram-se sempre associadas às partes coloridas das plantas, 
indicando que fatores físico-químicos incomuns devem estabilizá-las naturalmente (KORZUCHOWSKA e WILSKA, 1996; OSAWA 1982; ASEN, STEWART e NORRIS, 1972). Um desses fatores pode ser a presença de compostos chamados copigmentos, tais como flavonóides não-antociânicos, alcalóides, aminoácidos e nucleosídeos, entre outros (KORZUCHOWSKA e WILSKA, 1996; OSAWA, 1982).

A copigmentação pode ocorrer intra ou intermolecularmente (MARKAKIS, 1982) e sendo fenômenos com a mesma natureza, ambos competem pelo meio vacuolar das plantas (ELHABIRI, 2001). Fatores como o etanol, luz, pH, temperatura, estruturas e concentrações do copigmento e da antocianina, entre outros, influenciam significativamente a reação de copigmentação.

A reação de copigmentação relatada por WILLSTATTER e ZOLLINGER foi posteriormente estudada por ROBINSON e ROBINSON (1931). Esses avaliaram o efeito de diferentes copigmentos e sua habilidade em conferir desvios para a tonalidade azul às soluções acidificadas de "oenina" (malvidina 3-glucosídio). Perceberam a formação de complexo que se dissociava em altas temperaturas e na presença de determinados solventes extratores.

Diferentes técnicas têm sido utilizadas por pesquisadores para estudar a reação de copigmentação de antocianinas, como as de Espectroscopia de Ressonância Magnética Nuclear (H-NMR), Ramam, Massa e Ultravioleta-Visível (UV-Visível), dicroísmo circular (CD) e métodos microespectrofotométricos, dentre outras. A técnica de espectroscopia UV-Visível é a ferramenta mais popular para investigação da reação de copigmentação, sendo utilizada principalmente na pesquisa de compostos já relatados estruturalmente, e em estudos cinéticos e termodinâmicos.

\subsection{COPIGMENTAÇÃO INTRAMOLECULAR DE ANTOCIANINAS}

A copigmentação intramolecular ocorre somente quando o pigmento e o copigmento fazem parte da mesma molécula, ou seja, quando o cromóforo da antocianina e o copigmento (resíduo de ácido cinâmico) estão covalentemente ligados ao mesmo resíduo de açúcar (Figura 2) (BROUILLARD, 1983). Seu efeito é mais eficiente na estabilização 
das antocianinas do que a copigmentação intermolecular. Sob o ponto de vista termodinâmico apresenta vantagem entrópica sobre o efeito intermolecular (FRANCIS, 1989), uma vez que não é necessário unir as moléculas inicialmente separadas em solução (BROUILLARD, 1983). Os resíduos aromáticos de grupos acil alinham-se com o cátion flavilium dificultando a adição da água nas posições 2 e 4 do anel pirilium. Dessa forma, a dificuldade na hidratação da molécula e o aparente impedimento das reações de transferência de prótons pelo processo de "empilhamento" das moléculas aumentam sensivelmente a estabilidade dos cromóforos. É provável que as forças que atuam nesse processo sejam hidrofóbicas (BROUILLARD, 1983; MAZZA e MINIATI, 1993).

\section{FIGURA 2 - MODELO HIPOTÉTICO DA COPIGMENTAÇÃO INTRAMOLECULAR DE ANTOCIANINAS}

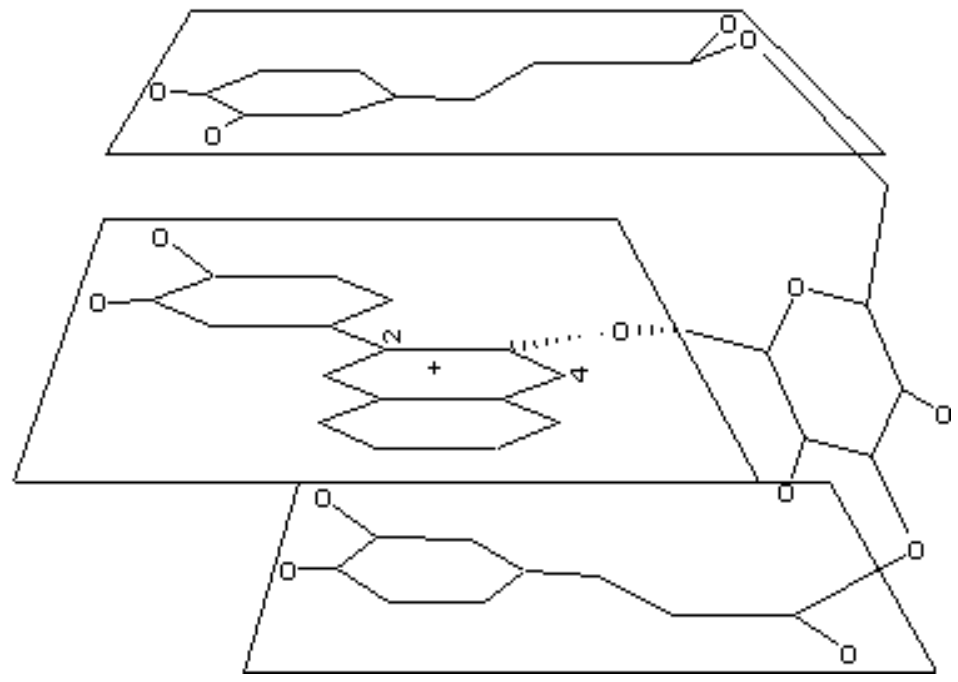

Fonte: BROUILLARD, 1983.

A maioria dos pigmentos acilados apresenta resíduos de ácidos cinâmico e/ou malônico esterificados aos açúcares. A cadeia flexível de sacarídios age como ligante, permitindo o dobramento dos anéis aromáticos sobre a estrutura planar do anel pirilium (FIGUEIREDO et al., 1996). 
A copigmentação intramolecular é possivelmente responsável pela extraordinária estabilidade dos cromóforos de antocianinas poliaciladas (BROUILLARD, 1983; MAZZA e MINIATI, 1993). A coloração da zebrina, extraída da Zebrina pendula (GOTO e KONDO, 1991) é exclusivamente produzida pelo pigmento em si, sem nenhuma interferência de outros agentes. A pouca estabilidade das antocianinas monoaciladas indica que somente um lado do anel pirilium é efetivamente protegido, o que permite a ação da água (HOSHINO, MATSUMOTO e GOTO, 1980).

Diversas pesquisas estão sendo realizadas a fim de estudar detalhadamente o comportamento da reação de copigmentação de antocianinas. FIGUEIREDO et al. (1996), utilizando espectroscopia UV-Visível e H'NMR, investigaram o mecanismo de copigmentação intramolecular de antocianinas e desenvolveram modelo matemático para relatar as propriedades corantes exibidas por antocianinas extraídas de flores violetas (Mathiola incana). Os resultados obtidos supõem a existência de interações intramoleculares não-covalentes que fortemente estabilizam as formas coloridas das antocianinas.

Antocianinas aciladas são mais estáveis do que as não-aciladas frente à degradação térmica. DYRBY, WESTERGARD e STAPELFELDT (2001) investigaram a estabilidade térmica de diferentes extratos de antocianinas em sistemas tampão pH 3,0 e temperaturas entre $25^{\circ} \mathrm{e}$ $80^{\circ} \mathrm{C}$, incluindo o extrato de repolho roxo (Brassica oleraceae L.) (no qual as antocianidinas cianidina-3,5-diglicosídio e cianidina-3sorofosídio-3-glicosídio, aciladas com os ácidos cinâmico, ferúlico, $p$ cumárico, caféico ou malônico, são predominantes). Os resultados indicaram maior estabilidade à degradação térmica dos extratos de repolho roxo, blackcurrant, uva e elderberry, respectivamente. A excelente estabilidade das antocianinas de repolho roxo se deve às moléculas ricas em copigmentos que protegem o cátion flavilium do ataque nucleofílico da água. Em contraste, os extratos de blackcurrant, uva e elderbverry não apresentam antocianinas aciladas com ácidos aromáticos.

A estabilidade de antocianinas não-aciladas foi demonstrada no trabalho de SARNI-MANCHADO et al. (1996), no qual o extrato bruto de uva (Vitis vinifera var. Grenache noir) foi purificado e analisado por Cromatografia a Líquido de Alta Eficiência (CLAE) fase-reversa. O estudo da estabilidade da fração, contendo malvidina 3-glicosídio, foi realizado 
com solução tampão pH 3,0 e 5,0, na presença de oxigênio e ausência de luz a 55ㄷ․ Após 8 dias foi verificada redução de $80-90 \%$ da cor inicial das soluções, indicando pouca estabilidade.

A presença de ácido caféico na molécula aumenta a estabilidade de antocianinas. DANGLES, SAITO e BROUILLARD (1993) sugeriram a existência de interação entre o cromóforo de pelargonidina e grupos cafeoil de antocianinas extraídos de pétalas de Pharbits nil (cultivares vermelho-púrpura). Utilizando H-NMR, espectroscopia de Massa e espectroscopia UV-Visível, verificaram que a cafeilação de agliconas pelargonidina diminui a constante termodinâmica de hidratação, retardando o deslocamento do equilíbrio da forma cátion flavilium (vermelho) para hemiacetal (incolor). Os pigmentos tri, di e monocafeilados apresentaram maior estabilidade na ordem citada. Foi demonstrado também, que antocianinas 3,5-diglicosídios foram mais rapidamente hidratadas do que as correspondentes 3-monoglicosídios.

Outros experimentos têm sido realizados a fim de investigar a acilação do cromóforo pelargonidina. GIUSTI, SAONA-RODRIGUEZ e WROLSTAD (1999) observaram os efeitos da glicosilação e da acilação sobre as características espectrais, absortividade molar e atributos de cor de pelargonidina acilada e não-acilada. Os pigmentos foram extraídos de diferentes fontes (morangos, radishes, batata vermelha) $\mathrm{e}$ isolados por CLAE. Foi verificado coeficiente de absortividade menor para a aglicona e pelargonidina-3-glicosídio em relação à pelargonidina5-glicosídio ou pelargonidina-3,5-glicosídio. A análise de cor foi realizada utilizando colorímetro de Hunter ColorQuest. A acilação com diferentes ácidos cinâmicos ( $p$-cumárico e malônico e somente $p$ cumárico) demonstrou efeitos consideráveis sobre as características espectrais e de cor. O solvente influenciou significativamente as características de cor de todos os derivados de pelargonidina, sendo que o metanol conferiu não somente efeito hipercrômico (com maior valor chroma), mas também produziu valores hue menores. A natureza do açúcar e as diferentes posições em que o ácido cinâmico se liga ao açúcar são fatores capazes de conferir características de cores diferentes à molécula de antocianina.

Pelargonidina-3-glicosídio, malvidina 3,5-diglicosídio e a monardeína (pelargonidina 3,5 diacilada com os ácidos cumárico e malônico) extraídas de pétalas de Monarda fistulosa foram investigadas quanto 
à capacidade de copigmentação com rutina e com os ácidos clorogênico e caféico (DAVIES e MAZZA, 1993). O efeito da copigmentação foi maior para monardeína do que para a correspondente pelargonidina-3-glicosídio. $\mathrm{O}$ pH influenciou significativamente a reação, sendo o efeito máximo da copigmentação observado entre pH 3,2-3,7 para o ácido clorogênico e entre 3,5-4,7 para o ácido caféico e a rutina. Os três compostos fenólicos reagiram de forma diferente para cada pigmento estudado, indicando que as estruturas do pigmento e do copigmento influenciam a magnitude da reação de copigmentação. Comparando os complexos formados entre os copigmentos e os pigmentos malvina e monardeína foi observado que os substituintes acil e metil influenciam significativamente a copigmentação. A presença de grupos metil na molécula de malvidina 3-monoglicosídio conferiu efeito de copigmentação maior do que a substituição com diacil da monardeína.

\subsection{COPIGMENTAÇÃO INTERMOLECULAR DE ANTOCIANINAS}

Na complexação intermolecular (Figura 3) predominam, provavelmente, forças de Van der Waals e efeitos hidrofóbicos em meio aquoso como resultado do "empilhamento" entre a molécula de antocianina e o copigmento (DANGLES, WIGAND e BROUILLARD, 1992).

Antocianinas, quando hidratadas, são convertidas à forma pseudobase incolor (Figura 1). A reação de copigmentação estabiliza as antocianinas em suas formas coloridas e retarda a reação normal de hidratação (BROUILLARD, 1983; SARNI-MANCHADO et al., 1996). Por isso, assume-se que a copigmentação constitua processo seletivo das formas coloridas (BROUILLARD et al., 1989).

O aumento da estabilidade ocorre porque o copigmento compete com a água e interage com as antocianinas, complexando as formas coloridas e modificando a natureza do copigmento (MAZZA e MINIATI, 1993). Certos copigmentos provocam aumento nos valores de absorvância (efeito hipercrômico) e deslocamento batocrômico (geralmente entre 5 e $20 \mathrm{~nm}$ ou mais no comprimento de onda de máxima absorção) (BOULTON, 2001; MAZZA e MINIATI, 1993; BROUILLARD, 1983; TIMBERLAKE, 1980), evidenciando a reação de copigmentação. Tais efeitos conferem tonalidade azul-púrpura às 
soluções vermelhas de antocianinas (BOULTON, 2001) e refletem satisfatoriamente a estabilidade do complexo formado (BROUILLARD, 1983). ASEN, STEWART e NORRIS (1972) observaram deslocamento batocrômico de $20 \mathrm{~nm}$ e aumento de $228 \%$ na absorção máxima da região visível em sistema composto de cloreto de cianina adicionado de rutina em $\mathrm{pH} 3,32$.

FIGURA 3 - COPIGMENTAÇÃO INTERMOLECULAR (1:1) ENTRE
APIGENIDINA E QUERCETINA-5'- ÁCIDO SULFÔNICO

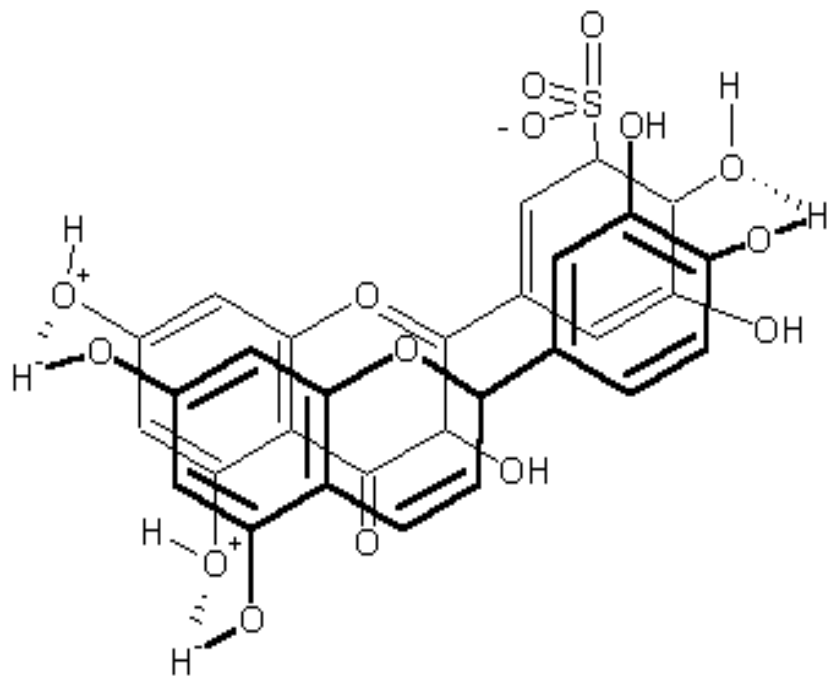

Fonte: IACOBUCCI e SWEENY, 1983.

O efeito hipercrômico não ocorre devido ao aumento do coeficiente de absorção, mas ao aumento da concentração de moléculas coloridas. O efeito batocrômico pode ser explicado pela redução local na polaridade do cromóforo flavilium, causado pelo envolvimento desse com o copigmento mediante associações hidrofóbicas (DANGLES, SAITO e BROUILLARD, 1993; BROUILLARD et al., 1989). O complexo pigmento-copigmento formado depende da concentração de ambos 
os compostos. Elevando-se a concentração desses, o efeito hipercrômico e o deslocamento batocrômico no comprimento de onda de máxima absorção também aumentam (BROUILLARD, 1983; OSAWA, 1982).

A presença de etanol reduz o efeito da copigmentação de antocianinas. Em estudo realizado com extratos de morango e chokeberry adicionados de ácido clorogênico foi verificado que 5\% de etanol (utilizado como co-solvente) diminuiu o efeito da interação do ácido com os pigmentos antociânicos. Em pH 3,4, a presença de etanol (10\%) resultou na diminuição de $20-25 \%$ nos valores de absorvância de soluções de antocianina:ácido clorogênico (1:25) (KORZUCHOWSKA e WILSKA, 1996).

MINIATI, DAMIANI e MAZZA (1992) utilizaram 3,5- diglucosídios de cianidina, pelargonidina e malvidina adicionados de ácido gálico, quercetina e catequina em $\mathrm{pH}$ 2,5, 3,5 e 4,5 para avaliar sua estabilidade em solução aquosa e de etanol 10\%. A adição de quercetina às soluções aumentou as absorvâncias em $47 \%$ para pelargonidina, $4 \%$ para malvina e 1\% para cianidina. Em presença de etanol, a reação de copigmentação foi fortemente afetada. O ácido gálico e a quercetina mostraram efeito superior à catequina quanto ao aumento da estabilidade das antocianinas. O copigmento mais eficiente foi a quercetina, pois a concentração utilizada era três vezes menor do que a do ácido gálico.

O extrato bruto de antocianinas de jambolão (Eugenia jambolona Lamark) foi avaliado por MARQUES, FETT e BRIGHENTE (2001). Esses analisaram a influência dos ácidos tânico e gálico (diferentes proporções) em soluções alcoólica e aquosa com pH 2,0. Paralelamente, avaliaram a estabilidade das antocianinas em presença dos ácidos salićlico, ftálico, cítrico e benzóico em solução alcoólica. Todas as soluções foram investigadas a $10^{\circ} \mathrm{C}$ e $30^{\circ} \mathrm{C}$. O aumento da temperatura, a presença de luz e a presença de álcool influenciaram significativamente a degradação das antocianinas, mesmo quando adicionadas de ácidos orgânicos (tânico e gálico). A adição de ácido salicílico e ácido gálico, em solução alcoólica e aquosa, conferiu aumento na capacidade de retenção de cor das antocianinas.

O extrato bruto de repolho roxo (Brassica oleraceae L.) adicionado de 
ácido tânico, em pH 3,0 e temperaturas de $4^{\circ} \mathrm{C}\left( \pm 1^{\circ} \mathrm{C}\right)$ e $30^{\circ} \mathrm{C}\left( \pm 2^{\circ} \mathrm{C}\right)$, foi avaliado por FALCÃO et al. (2002). Em comparação ao controle observaram aumento de 245 e 190 horas no tempo de meia-vida das amostras com ácido tânico a $4^{\circ} \mathrm{C}$ e $30^{\circ} \mathrm{C}$, respectivamente. Em experimentos com vinho tinto, DARÍAS-MARTíN et al. (2001) verificaram o efeito da adição de ácido caféico e catequina ao mosto do vinho na fase de pré-fermentação. Após 90 dias, verificaram que o ácido caféico conferiu aumento de $60 \%$ nos valores de absorvância do vinho, enquanto que o vinho adicionado com catequina apresentou valores de absorvância inferiores ao controle.

Antocianinas do extrato bruto de laranjas pigmentadas foram avaliados em diferentes valores de $\mathrm{pH}$ e temperaturas em sistemas reais de alimentos, simulando bebidas carbonatadas e alcoólicas (KATSABOXAKIS, PAPANICOLAOU e MELANITOU, 1998). Em pH 2,5 e a $80^{\circ} \mathrm{C}$ o tempo de meia-vida das antocianinas foi de 791 minutos (13, 2 h), enquanto que em $\mathrm{pH}$ 4,5 foi de 197 minutos (3,3 h). Quando aquecidas em temperaturas de $60^{\circ} \mathrm{C}, 70^{\circ} \mathrm{C}$ e $80^{\circ} \mathrm{C}$ a diminuição de cor do extrato antociânico em pH 3,5 foi inferior a $20 \%$, enquanto que a $90^{\circ} \mathrm{C}$ e $100^{\circ} \mathrm{C}$ foi superior a $50 \%$. A estabilidade desse extrato em bebidas carbonatadas $(\mathrm{pH} 3,0)$ foi avaliada a $4^{\circ} \mathrm{C}$ e $30^{\circ} \mathrm{C}$. Após sete meses, foi verificada perda de $50 \%$ da cor nas amostras mantidas a $30^{\circ} \mathrm{C}$ e de apenas $30 \%$ nas amostras que permaneceram a $4^{\circ} \mathrm{C}$. O efeito da luz acelerou a redução da cor de bebidas etanólicas adicionadas de extrato bruto de laranja. Após três meses, tal descréscimo situou-se em torno de $20 \%$ nas amostras mantidas sob ausência de luz, enquanto que as amostras expostas à luz perderam cerca de $50 \%$ da cor.

Experimentos simulando sistemas modelos de alimentos são importantes sob o ponto de vista prático para avaliação do comportamento das antocianinas e para potencializar sua aplicação. Porém, estudos visando a verificação da estabilidade da reação de copigmentação de antocianinas em sistemas que imitam a composição de alimentos ainda são necessários.

\section{CONCLUSÃO}

A reação de copigmentação, inter e intramolecular, aumenta a 
estabilidade das antocianinas e, com isso, seu potencial de aplicação. Estudos para avaliar a estabilidade de antocianinas adicionadas de copigmentos em sistemas modelos de alimentos ainda são necessários, visando aumentar o espectro de aplicação dessas como corantes em alimentos e bebidas.

\section{Abstract}

\section{ANTHOCYANINS INTRA AND INTERMOLECULAR COPIGMENTATION: A REVIEW}

This research presents literature review about the reaction of intra and intermolecular copigmentation and its revealance in anthocyanins stabilization. Non-anthocyanic flavonoids, alkaloids, amino acids, nucleosides, and others, can act as anthocyanins copigments. The increase in the stability of anthocyanins occurs due to protection supplied by the copigment towards the hydratation reaction of colored flavylium cation. Studies to evaluate anthocyanins stabilization added of copigments in food models system are still necessary, aiming to enhance the application spectra as colorants in foods and beverages.

KEY-WORDS: COPIGMENTATION; ANTHOCYANINS.

\section{REFERÊNCIAS}

1 ASEN, S.; STEWART, R.N.; NORRIS, K.H. Co-pigmentation of anthocyanins in plant tissues and its effect on color. Phytochemistry, v.11. n. 3, p. 1139-44, 1972.

2 AUBERT, C. M.; DANGLES, A.; AMIOT, M. J. Color stability of commercial anthocyanin-based extracts in relation to the phenolic composition. Protective effects by intra and intermolecular copigmentation. Journal Agricultural Food Chemistry, v. 49, p. 170-176, 2001.

3 BARANAC, J. M.; PETRANOVIC, N. A.; DIMITRIC-MARKOVIC, J.M. Spectrophometric study of anthocyan copigmentation reactions. Journal Agricultural Food Chemistry, v. 44, p. 1333-1336, 1996.

4 BOULTON, R. The copigmentation of anthocyanins and its role in the color of red wine: A Critical Review. American Journal of Enology and Viticulture, v. 52, n. 2, p. 67-80, 2001.

5 BROUILLARD, R. The in vivo expression of anthocyanin colour in plants. Phytochemistry, v. 22, n. 6, p. 311-23, 1983. 

copigmentation reaction of anthocyanins: A micropobe for the structural study of aqueous solutions. Journal American Chemical Society, v. 111, p. 247-2, 1989.

7 DANGLES, O; WIGAND M. C.; BROUILLARD, R. Anthocyanin anti-copigment effect. Phytochemistry, v. 31, n. 11, p. 3811-3812, 1992.

8 DANGLES, O.; BROUILLARD, R. A spectrophotometric method based on the anthocyanin copigmentation interaction and applied to the quantitative study of molecular complexes. Journal Chemical Society Perkin Trans 2, v. 2, p. 247-257, 1992.

9 DANGLES, O; SAITO, N.; BROUILLARD, R. Anthocyanin Intramolecular copigment effect. Phytochemistry, v. 34, n 1, p. 119-124, 1993.

DARÍAS-MARTÍN, J. et al. Enhancement of red wine colour by pre-fermentation addition of copigments. Food Chemistry, v. 73, p. 217-220, 2001.

11 DAVIES, A. J.; MAZZA, G. Copigmentation of simple acylated anthocyanins with colorless phenolic compounds. Journal Agricultural Food Chemistry, v. 41, n. 5, p. 716-720, 1993.

12 DIMITRIC-MARKOVIC, J. M. D.; PETRANOVIC, N. A.; BARANAC, J. M. Spectophotometric study of the copigmentation of malvin with caffeic and ferulic acids. Journal Agricultural Food Chemistry, v. 48, p. 5530-5536, 2000.

13 DYRBY, M.; WESTERGARD, N.; STAPELFELDT, H. Light and heat sensitivity of red cabbage extract in soft drink models systems. Food Chemistry, v. 72, p. 431-437, 2001.

14 ELHABIRI, M. Les poliphénols: présentation de travail de thèse. Disponível em: <http://membres.lycos.fr/mourad/essai.html>. Acesso em_08/10/2001.

FALCÃO, L. D.; GAUCHE, C.; BARROS; D. OGLIARI, P.; FETT, R. LUIZ, M. T. B. Estudo da estabilidade de antocianinas de repolho roxo (Brassica oleraceae L.) adicionadas de ácido tânico. In: CONGRESSO BRASILEIRO DE CIÊNCIA E TECNOLOGIA DE ALIMENTOS, 18., Porto Alegre, RS, 2002. Anais... Porto Alegre: Sociedade Brasileira de Ciência e Tecnologia de Alimentos, 2002. p. 3055-3058.

16 FIGUEIREDO, P.; ELHABIRI, M.; SAITO, N.; BROUILLARD, R. Anthocyanin intramolecular interactions: a new mathematical approach to account for the remarkable colorant properties of the pigments extracted from Matthiola incana. Journal Agricultural Food Chemistry, v. 118, p. 4788-4793, 1996. 
17 FRANCIS, F. J. Food colorants: anthocyanins. Food Science and Nutrition, v 28, p. 273-314, 1989.

18 GIUSTI, M.; SAONA-RODRIGUEZ, L.E.; WROLSTAD, R. E. Molar absortivity and color characteristics of acylated and non-acylated pelargonidin-based anthocyanins. Journal Agricultural Food Chemistry, v. 47, p. 4631-4637, 1999.

19 GONNET, J. F. Colour effects of co-pigmentation of anthocyanins revisited-1. A colorimetric definition using the CIELAB scale. Food Chemistry, v. 63, n. 3, p. 409-4015, 1998.

20 GOTO, T., KONDO, T. Structure and molecular staking of anthocyanins flower color variation. Angew. Chem. Int. Ed. Engl., v. 30, p. 17-33, 1991.

21 HAGIWARA, A. et al. Pronounced inhibition by a natural anthocyanin, purple corn color, of 2-amino-16-phenylimidazol $(4,5-b)$ pyridine (PhIP)-associated colorectal carcinogenesis in male F344 rats pretreated with 1,2dimethylhydrazine. Cancer Letters, v. 171, p. 17-25, 2001.

22 HOSHINO, T.; MATSUMOTO, U.; GOTO, T. The stabilizing effect of the acyl group on the copigmentation of acyled anthocyanins with C-glucosylflavones. Phytochemistry, v. 19, p. 663, 1980.

23 IACOBUCCI, G. A.; SWEENY, J. G. The chemistry of anthocyanins, anthocyanidins and related flavilium salts. Tetrahedron Letters, v. 39, n. 19, p. 3005-30038. 1983.

24 KAPADIA, G. J.; BALASUBRAMANIAN, V.; TOKUDA, H.; I WASHINA, A; NISHINO, H. Inhibition of 12-O-tetradecanoylphorbol-13-acetate induced Epstein virus early antigen activation by natural colorants. Cancer Letters, n. 115, p. $173-178,1997$.

25 KATSABOXAKIS, K.; PAPANICOLAOU, D.; MELANITOU, M. Stability of pigmented orange anthocyanins in model and real food system. Italian Journal Food Science, v. 10, n. 1, p. 17-25, 1998.

26 KORZUCHOWSKA, A. e WILSKA J. J. Anthocyanins and chlorogenic acid copigmentation: influence on the colour of strawberry and chockeberry juices. Lembesm Unters Forsch, v. 203. n. 1, p. 38-42, 1996.

27 MARKAKIS, P. Anthocyanin as food colors. New York: Academic Press, 1982.

28 MARQUES, P.; FETT, R.; BRIGHENTE, I. Influência da copigmentação no extrato bruto do jambolão (Eugenia jambolona LamarK). In:SIMPÓSIO EM CIÊNCIA DE ALIMENTOS, ALIMENTOS \& SAÚDE, QB 36, 07 de junho de 2001, Florianópolis, SC. Anais... Florianópolis: Universidade Federal de Santa Catarina, 2001. p. 141 . 
MAZZA, G.; BROUILLARD, R. Recent developments in the stabilization of anthocyanins in food products. Food Chemistry, v. 25, p. 207-225, 1987.

30 MAZZA, G. e MINIATI, E. Anthocyanins in fruits, vegetables and grains, Boca Raton-Florida (USA): CRC Press. 1993.

31 MINIATI, E.; DAMIANI, P.; MAZZA, G. Copigmentation and self-assocciation of anthocyanins in food model sistems. Italian Journal Food Science, v. 4, n. 2, p. 109-116, 1992.

32 OSAWA, Y. Copigmentation of anthocyanins. In:MARKAKIS, P. Anthocyanins as food colors. New York: Academic Press, 1982.

33 PROVENZI, G. Estabilidade de enocianinas adiconadas de $\alpha, \beta$ e $\gamma$ ciclodextrina e aplicação em gelatina. Florianópolis, 2001. $73 \mathrm{f}$. Dissertação (Mestrado em Ciência de Alimentos), Universidade Federal de Santa Catarina.

34 ROBINSON, G. M.; ROBINSON, R. A survey of anthocyanins. The Biochemical Journal, v. 2, p. 1687-1705, 1931.

35 SAONA-RODRIGUEZ, L. E.; GIUSTI, M.; WROLSTAD, R. E. Anthocyanin pigment composition of red-fleshed potatoes. Journal of Food Science, v. 63, p. 458-465, 1998.

36 SARNI-MANCHADO, P. S.; FULCRAND, H.; SOUQUET, J.M.; CHEYNIER, V.; MOUTOUNET, M. Stability and color of unreported wine anthocyanin-derived pigments. Journal of Food Science, v. 61, n. 5, p. 938-941, 1996.

37 STRINGHETA, P. C. Identificação da estrutura e estudo da estabilidade das antocianinas extraídas da influorescência de capim gordura (Mellinis minutiflora, Pal de Beauv). São Paulo, 1991. 138 f. Tese (Doutorado em Ciência de Alimentos). Faculdade de Engenharia de Alimentos, Universidade Estadual de Campinas.

38 TAMURA, H.; TAKADA, M.; YAMAGAMI, A; SHIOMI, K. The color stability and antioxidative activity of an anthocyanin and gamma-cyclodextrin complex. In: TAKAYUKI, S.; JUNJI, T.; TOSHIHIKO, O. Functional foods for disease prevention. I. Fruits, vegetables, and teas. Oxford: University Press, 1998. Chapter 16, p. 157-171.

39 TIMBERLAKE, C.F. Anthocyanins occurrence, extraction and chemistry. Food Chemistry, Great Britain, n. 5, p.69-80, 1980.

40 WANG, C. J.; WANG, J. M.; WEA, L. L.; CHIA, Y. C.; CHOU, F. P.; TSENG, T. H. Protective effect of Hibiscus Anthocyanins Against tert-butyl Hidroperoxideinduced Hepatic Toxicity in Rats. Food and Chemical Toxicology, v. 38, p. 411-416, 2000. 
41 YOUDIM, K. A.; MARTIN, A.; JOSEPH, J. A. Incorporation of elderberry anthocyanins by endothelial cells increases protection against oxidative stress. Free Radical Biology \& Medicine, v. 29, n. 1, p. 51-60, 2000. 